\title{
Halbach undulators using right triangular magnets
}

\author{
N. Majernik $\oplus^{*}$ and J. B. Rosenzweig \\ University of California - Los Angeles, Los Angeles, California 90095, USA
}

(Received 23 July 2019; published 26 September 2019)

\begin{abstract}
Undulators and wigglers based on Halbach arrays have been in widespread use since their description in the 1980s, due to the high fields they generate from permanent magnets. The most common implementation of a pure permanent magnet Halbach undulator consists of four magnetic segment widths per undulator period. In typical undulators with periods of order $\sim \mathrm{cm}$ it is straightforward to use sufficiently small magnetic segments to achieve the desired undulator period. However, in the context of new microundulators with periods of order $\sim \mathrm{mm}$ or shorter, there is a lower bound on the feature sizes achievable by existing fabrication techniques. This motivates the use of modified Halbach arrays comprised of right triangle magnet segments with only two magnet segment widths per undulator period. These configurations are demonstrated to have superior field strengths to up-down arrays with no increase to the undulator period. Additional considerations arising from the broken symmetries in such configurations, such as the presence of previously forbidden harmonic components, are also considered. Such right triangle Halbach arrays may be fabricated using MEMS techniques for use in the next generation of compact light sources.
\end{abstract}

DOI: 10.1103/PhysRevAccelBeams.22.092401

\section{INTRODUCTION}

The Halbach array of magnets, described in $[1,2]$ by the eponymous Klaus Halbach, consist of arrays of magnets with smoothly rotating residual field orientations for the construction of multipole magnets and undulators. In the intervening decades, Halbach arrays have been used extensively in the construction of permanent magnet (PM) wigglers and undulators [3-6], enhanced multipole magnets including dipoles [7] and quadrupoles [8], in addition to use in motor design $[9,10]$ and other engineering applications [11]. The benefit of this configuration is the establishment of a "strong" and "weak" side to the array, whereby the field is enhanced on the strong side and attenuated on the weak side. This leads to more efficient use of the available magnetic flux, giving stronger fields than other configurations. Many variations exist, most notably the hybrid Halbach which includes both hard and soft ferromagnetic material $[12,13]$, which is capable of producing stronger fields through the use of high saturation yoke material. Despite this, the pure PM Halbach consisting only of hard ferromagnets is still commonly used.

There is growing interest in short period undulators (microundulators) [13-16], facilitated by MEMS

\footnotetext{
*NMajernik@g.ucla.edu

Published by the American Physical Society under the terms of the Creative Commons Attribution 4.0 International license. Further distribution of this work must maintain attribution to the author(s) and the published article's title, journal citation, and DOI.
}

(microelectromechanical systems) and other modern fabrication techniques, with researchers seeking to decrease the period length to achieve concomitant reductions in freeelectron laser (FEL) or light source size. In this context though, conventional Halbach undulators suffer from a distinct disadvantage: for a given feature size achievable by a particular machining or fabrication process, a conventional Halbach array will have twice the period of a simple up-down lattice $\left(M^{\prime}=2\right.$ in the terminology of [1]). This limitation motivates this exploration of triangle based Halbach arrays. The triangle Halbach involves less magnetic material per period than an $M^{\prime}=4$ undulator so lower fields are achieved, with the advantage that for a given feature size the undulator period is halved. Existing triangular-magnet Halbach arrays have relied on isosceles triangle geometries [17-19], leading to intrinsic symmetries.

Unfortunately, such arrays are difficult to fabricate, especially at microundulator length scales. To mitigate such challenges, simplification of the component magnets and their assembly must be reexamined. As such, here we discuss Halbach arrays based on right triangles, which feature easier fabrication while maintaining comparable performance to the isosceles case and superior performance to $M^{\prime}=2$ arrays. Fabrication might begin with bar magnets, manufactured to a high degree of accuracy, parallelism, and perpendicularity using standard, scalable machining processes like surface grinding. By using a through-bulk cutting technique like electrical discharge machining or laser machining along the hypotenuse a right angle triangle prism can be produced that is intrinsically indexed to the remaining, square section (Fig. 1). These 


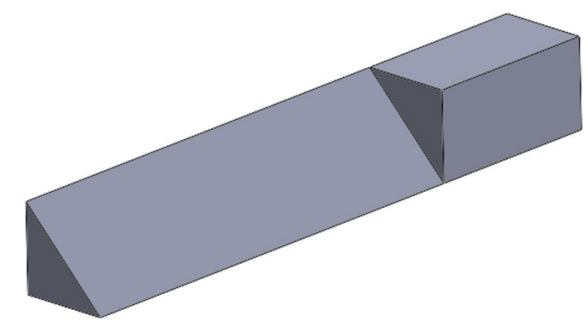

FIG. 1. An example right angle triangle magnet.

square parts can be indexed to each other and to flat surfaces very accurately, without relying on the nonground hypotenuse surfaces.

\section{SIMULATION SETUP}

\section{A. Overview}

Four configurations of magnets will be considered: the $M^{\prime}=2$ (up-down) array, isosceles triangles, reflection symmetric right triangles, and rotationally symmetric right triangles. Each case has an undulator period, $\lambda_{u}$, equal to twice the minimum feature size, and all cases have an equal volume of magnetic material per period, specifically each magnet has a height equal to half a period. These cases are shown in Fig. 2 along with the $M^{\prime}=4$ configuration, which has $\lambda_{u}$ equal to four times the minimum feature size. All simulations are performed using the magnetostatics code, RADIA [20] with the material properties of neodymium magnets.

\section{B. Magnetization vector}

The ideal magnetization vector as a function of longitudinal position, $z$, for a Halbach array [2] is simply $\hat{m}=$ $e^{i z k_{z}}$ where $k_{z}=2 \pi / \lambda_{u}$. In the case of undulators in actual experimental realization, however, the magnets are discrete elements, each with a discrete, constant magnetization. With $M^{\prime}$ square magnets per period each magnet's magnetization vector is rotated by a fixed amount from the previous one, meaning that the magnetization of the $n^{\text {th }}$ magnet is given by $e^{i 2 \pi n / M^{\prime}}$. This rule extends to the isosceles case, which exhibits the same rotation as $M^{\prime}=$ 4 arrays. However, applying this constant rotation to right angle arrays leads to suboptimal fields. Instead, the ideal vector field described by

$$
\hat{m}(z, y)=e^{i\left(z k_{z}+\phi\right)},
$$

where $\phi$ is a variable utilized to independently control the phase, is averaged over the region of each discrete magnet and normalized to one. For magnet $n=1$ [see Fig. 2(d)] this average vector is

$$
\langle\vec{m}\rangle_{n=1}=\frac{\int_{0}^{\lambda_{u} / 2} \int_{z}^{\lambda_{u} / 2} \hat{m}(z, y) \mathrm{d} y \mathrm{~d} z}{\lambda_{u}^{2} / 8},
$$

(a)

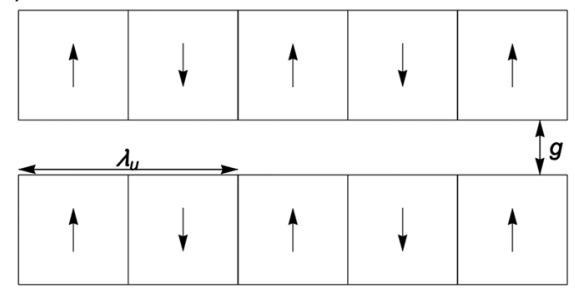

(b)
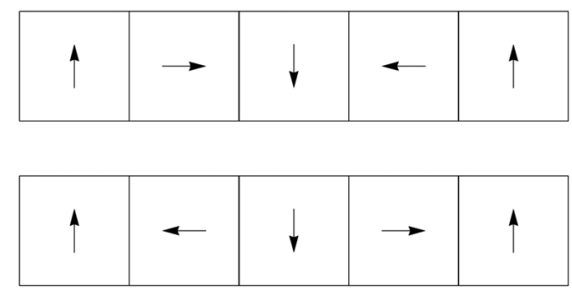

(c)
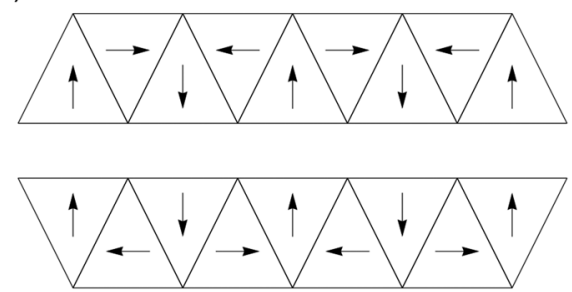

(d)
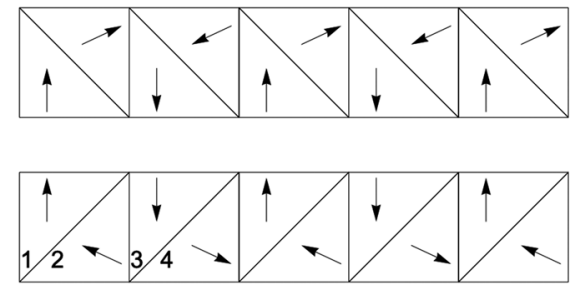

(e)
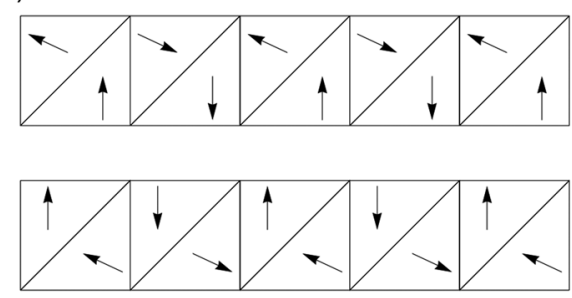

(f)
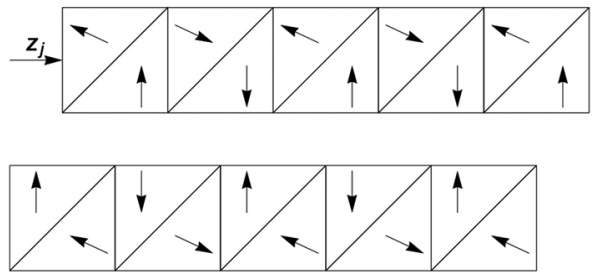

FIG. 2. Examples of the undulator configurations. (a) $M^{\prime}=2$ array, (b) $M^{\prime}=4$ array, (c) isosceles triangles, (d) reflection symmetric right triangles, (e) rotationally symmetric right triangles, and (f) rotationally symmetric right triangles with jaw translation (positive $z_{j}$ is illustrated but negative is typically used). 
TABLE I. RMS deviation between ideal Halbach magnetization and discrete magnet vector, by geometry.

\begin{tabular}{lc}
\hline \hline Geometry & RMS deviation \\
\hline$M^{\prime}=2$ & 0.109 \\
Right angle $\left(90^{\circ}\right)$ & 0.096 \\
Right angle (optimized) & 0.093 \\
Isosceles & 0.083 \\
$M^{\prime}=4$ & 0.062 \\
$M^{\prime} \rightarrow \infty$ & $\rightarrow 0$ \\
\hline \hline
\end{tabular}

which normalizes to the unit vector

$$
\begin{aligned}
\langle\hat{m}\rangle_{n=1} & =\frac{\langle\vec{m}\rangle_{n=1}}{\left|\langle\vec{m}\rangle_{n=1}\right|} \\
& =\frac{(2 \cos \phi-\pi \sin \phi) \hat{z}+(\pi \cos \phi+2 \sin \phi) \hat{y}}{\sqrt{4+\pi^{2}}} .
\end{aligned}
$$

The same approach for magnet $n=2$ yields

$$
\langle\hat{m}\rangle_{n=2}=\frac{-(2 \cos \phi+\pi \sin \phi) \hat{z}+(\pi \cos \phi-2 \sin \phi) \hat{y}}{\sqrt{4+\pi^{2}}} .
$$

The angle between these vectors is independent of $\phi$ and equal to $\pi-2 \tan ^{-1}(\pi / 2) \approx 64.96^{\circ}$. Magnets 3 and 4 have magnetization vectors $\langle\hat{m}\rangle_{n=3}=-\langle\hat{m}\rangle_{n=1}$ and $\langle\hat{m}\rangle_{n=4}=$ $-\langle\hat{m}\rangle_{n=2}$ respectively. Using the angles from this approach enhances the performance over the use of naive, $90^{\circ}$ rotations and is discussed in later sections. This approach can be extended to permit the calculation of the rms deviation between the discrete magnet's magnetization and the ideal field of Eq. (1), with these results shown in Table I (the rms deviation is independent of $\phi$ ). As will be shown later, this deviation is a predictor of the undulator strength.

\section{Impact of jaw translation}

The length scale is normalized to the period of the undulator, $\lambda_{u}$, leaving two free variables for each configuration: the gap, $g$, and the longitudinal translation between the jaws, $z_{j}$. The jaw gap is a commonly manipulated parameter for real world undulators: increasing the gap reduces the field on axis and also decreases the relative contributions of higher harmonics. Longitudinal translation is not often considered in the context of linear undulators, though it is employed in some adjustable helical undulators $[6,21,22])$. For existing undulators which are symmetric (i.e., $M^{\prime}=2, M^{\prime}=4$, and isosceles triangle) translations can serve to reduce the field strength at the cost of introducing errors [23]. As will be demonstrated below, however, for right triangle arrays, adjusting this parameter can be used to optimize the undulator's magnetic performance. All reflection symmetric cases maximize vertical field and minimize longitudinal fields at zero translation, but rotation symmetric undulators are optimized with a different, nonvanishing $z_{j}$, dependent on gap. Based on this study, a heuristic guide for the optimized translation is given by

$$
z_{j}\left[\lambda_{u}\right] \approx-0.025 \ln \left(g\left[\lambda_{u}\right]\right)-0.14
$$

This approximation gives a coefficient of determination ( $R^{2}$ value) of 0.9996 for values of $g$ ranging from $0.02 \lambda_{u}$ to $0.5 \lambda_{u}$.

\section{RESULTS}

\section{A. Strength comparison}

To compare between the different configurations, both the magnitude and quality of the on-axis fields will be considered. It is typical to characterize the strength of undulators and wigglers in terms of a unitless parameter called the $K$-value, sometimes also termed the strength parameter. This parameter is the normalized (to $m_{e} c$ ) vector potential, and thus is also equal in amplitude to the largest transverse momentum due to undulator-derived deflections. In this way, one sees that $K$ may be calculated by finding the maximum deflection angle induced by the undulator multiplied by the Lorentz factor, $\gamma$. Using the small angle approximation and neglecting nonvertical fields as in [21], the equation of motion for an electron in the oscillation plane is

$$
\frac{d^{2} x}{d s^{2}}=\frac{e}{\gamma m_{e} c} B_{y}(s),
$$

where $e$ is the electron charge, $m_{e}$ is the electron mass, $c$ is the speed of light, and $B_{y}(s)$ is the vertical field along the undulator. The maximum deflection angle and therefore the $K$-value is given by:

$$
\left.\frac{d x}{d s}\right|_{\max }=\frac{1}{2} \int_{0}^{\lambda_{u} / 2} \frac{e}{\gamma m_{e} c} B_{y}(s) \mathrm{d} s \equiv \frac{K}{\gamma} .
$$

For the idealized case of an undulator with a perfectly sinusoidal field, $B_{y}(s)=-B_{0} \sin \left(2 \pi s / \lambda_{u}\right)$, the $K$-value is

$$
K=\frac{B_{0} e \lambda_{u}}{2 \pi m_{e} c} \approx 93.36 B_{0}[T] \lambda_{u}[m] .
$$

Equation (7) will be used to calculate the relative strengths of different undulator configurations. However, $K$ depends on the magnetic material selected and $\lambda_{u}$ in absolute units so, to avoid confusion, $K$ will not be directly utilized in absolute scale, but instead the configurations will be compared in relative terms.

Over gap values ranging from $0.02 \lambda_{u}$ to $0.5 \lambda_{u}$ an isosceles array gives integrated fields approximately $19 \%$ higher than a $M^{\prime}=2$ array while optimized right triangle 
arrays (of both rotation and reflection symmetries) give integrated fields approximately $13 \%$ higher than an $M^{\prime}=2$ array. Using an unoptimized, $90^{\circ}$ magnetization vector rotation yields a $10 \%$ enhancement over $M^{\prime}=2$. This hierarchy of strength is consistent the rms deviations between the magnetization vector as compared with the ideal configuration detailed in Table I.

\section{B. Quality comparison}

The quality of the on-axis undulator field is sometimes described by taking a Fourier decomposition [24-26] and inspecting the harmonic components. In typical undulators, the even harmonics are negligible and the coefficients of the harmonics are only for the sine contribution. In the case of right-triangle arrays though, symmetries have been broken so, although the integrated field per period will always be zero (i.e., no net deflection of a beam) and while there are still no even harmonics, there may be non-negligible complex components. The coefficients for the first, third, and fifth harmonics are shown for $M^{\prime}=2$, isosceles triangle, rotation symmetric right triangle, and reflection symmetric right triangle arrays as functions of the gap in Fig. 3. It can be observed that, as noted, the first harmonic dominates at larger gap sizes at the cost of total field.
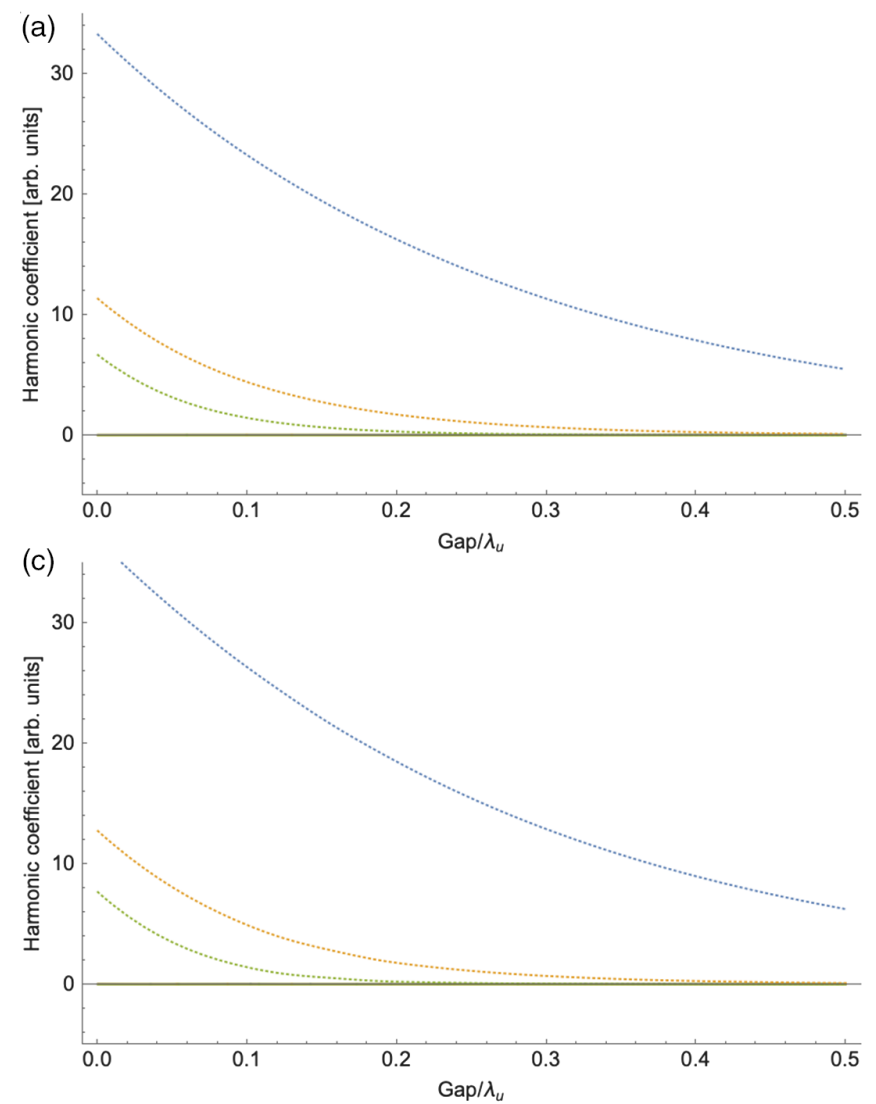

Such harmonics are not necessarily destructive and may perhaps even be valuable; previous research has used undulator harmonics to adjust the radiated spectrum [25] and to employ the inverse free-electron laser (IFEL) mechanism [27] at lower energy. With these issues in mind, $z_{j}$ was then varied to maximize higher harmonics rather than the fundamental. The results are similar to that of the fundamental, with the third and fifth harmonics of the isosceles array approximately $20 \%$ higher than the $M^{\prime}=2$ array and right triangle arrays approximately $14 \%$ higher than the $M^{\prime}=2$ array.

\section{CONCLUSIONS}

A new variant of the Halbach array undulator comprised of right triangle magnets has been described and compared to existing options including isosceles triangle arrays and up-down $\left(M^{\prime}=2\right)$ arrays. Such devices can offer integrated magnetic field strengths $13 \%$ higher than $M^{\prime}=2$ arrays with no increase in undulator period while remaining easier to fabricate than isosceles triangle arrays, which offer a $19 \%$ enhancement over $M^{\prime}=2$. These arrays lack some of the symmetries found in more conventional Halbach undulators, requiring the optimization of longitudinal jaw translation and introducing previously forbidden
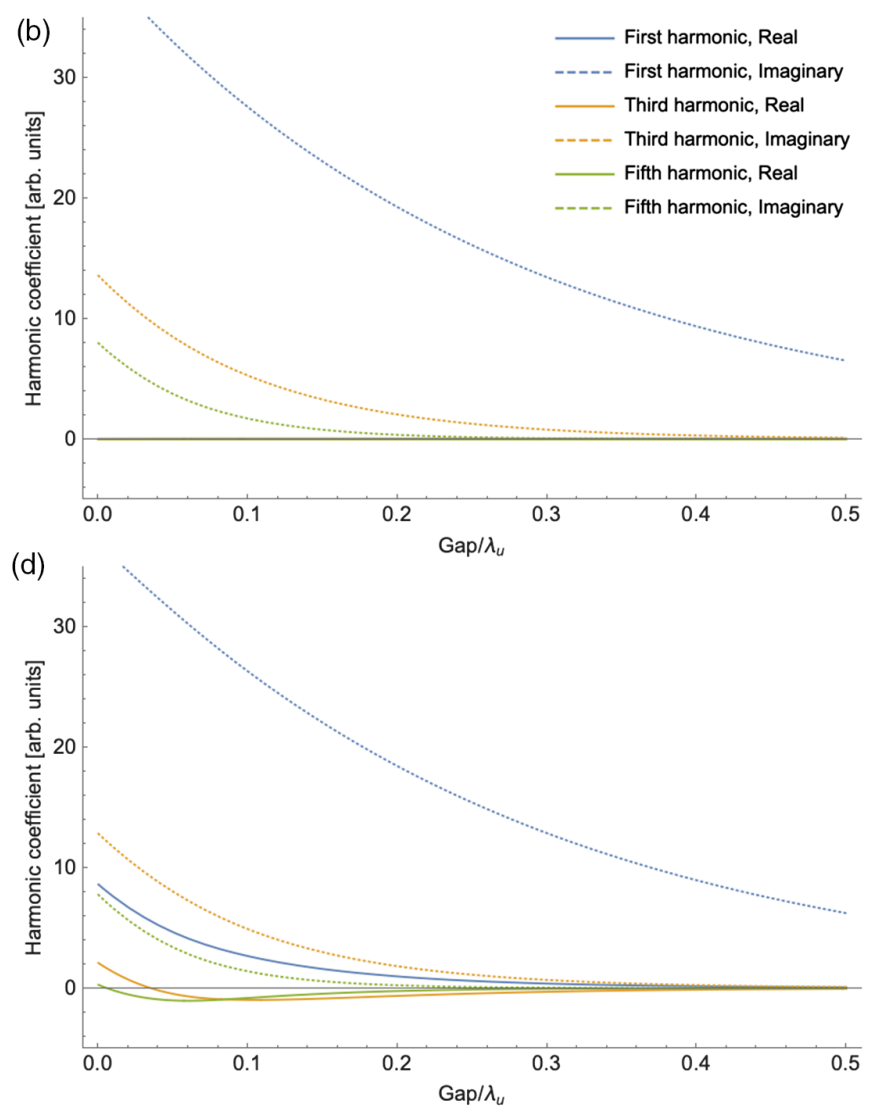

FIG. 3. Harmonic coefficients of the on axis field for (a) $M^{\prime}=2$, (b) isosceles triangle, (c) rotation symmetric right triangle, and (d) reflection symmetric right triangle. 
harmonic components. The use of these harmonics to tune the radiated spectrum or drive the IFEL mechanism means, in some cases, that higher harmonic content is desirable; these right triangle arrays similarly outperform standard $M^{\prime}=2$ arrays at the third order. These new configurations may be fabricated by today's MEMS manufacturing techniques to be used in the construction of the next generation of compact light sources [28].

\section{ACKNOWLEDGMENTS}

This work was supported by U.S. Department of Energy Contract No. DE-SC0009914 and by the U.S. NSF Grant No. PHY-1549132, the Center for Bright Beams.

[1] K. Halbach, Design of permanent multipole magnets with oriented rare earth cobalt material, Nucl. Instrum. Methods 169, 1 (1980).

[2] K. Halbach, Physical and optical properties of rare earth cobalt magnets, Nucl. Instrum. Methods 187, 109 (1981).

[3] R. Carr, M. Cornacchia, P. Emma, H. D. Nuhn, B. Poling, R. Ruland, E. Johnson, G. Rakowsky, J. Skaritka, S. Lidia, P. Duffy, M. Libkind, P. Frigola, A. Murokh, C. Pellegrini, J. Rosenzweig, and A. Tremaine, Visibleinfrared self-amplified spontaneous emission amplifier free electron laser undulator, Phys. Rev. Accel. Beams 4, 122402 (2001).

[4] A. Murokh et al., Results of the VISA SASE FEL experiment at $840 \mathrm{~nm}$, Nucl. Instrum. Methods Phys. Res., Sect. A 507, 417 (2003).

[5] K. Halbach, J. Chin, E. Hoyer, H. Winick, R. Cronin, J. Yang, and Y. Zambre, A permanent magnet undulator for SPEAR, IEEE Trans. Nucl. Sci. 28, 3136 (1981).

[6] S. Lidia and R. Carr, An elliptically-polarizing undulator with phase adjustable energy and polarization, Nucl. Instrum. Methods Phys. Res., Sect. A 347, 77 (1994).

[7] M. Kumada, Y. Iwashita, M. Aoki, and E. Sugiyama, in Proceedings of the 2003 Particle Accelerator Conference, Portland, OR (IEEE, New York, 2003), https://accelconf .web.cern.ch/accelconf/p03/PAPERS/WPAE024.PDF.

[8] J. K. Lim, P. Frigola, G. Travish, J. B. Rosenzweig, S. G. Anderson, W. J. Brown, J. S. Jacob, C. L. Robbins, and A. M. Tremaine, Adjustable, short focal length permanentmagnet quadrupole based electron beam final focus system, Phys. Rev. ST Accel. Beams 8, 072401 (2005).

[9] K. Atallah and D. Howe, A magnetic coupled charger with no-load protection, IEEE Trans. Magn. 34, 2057 (1998).

[10] Z. Zhu and D. Howe, Halbach permanent magnet machines and applications: A review, IEE Proc.-Electric Power Applications 148, 299 (2001).

[11] D. Zhu, S. Beeby, J. Tudor, and N. Harris, Increasing output power of electromagnetic vibration energy harvesters using improved Halbach arrays, Sens. Actuators, A 203, 11 (2013).
[12] P. Elleaume, J. Chavanne, and B. Faatz, Design considerations for a $1 \AA$ SASE undulator, Nucl. Instrum. Methods Phys. Res., Sect. A 455, 503 (2000).

[13] T. Eichner, F. Grüner, S. Becker, M. Fuchs, D. Habs, P. Kunz, R. Weingartner, H. Backe, U. Schramm, and W. Lauth, Miniature magnetic devices for laser-based, tabletop free-electron lasers, Phys. Rev. Accel. Beams 10, 082401 (2007).

[14] J. Harrison, A. Joshi, J. Lake, R. Candler, and P. Musumeci, Surface-micromachined magnetic undulator with period length between $10 \mu \mathrm{m}$ and $1 \mathrm{~mm}$ for advanced light sources, Phys. Rev. Accel. Beams 15, 070703 (2012).

[15] G. Ramian, L. Elias, and I. Kimel, Micro-undulator FELS, Nucl. Instrum. Methods Phys. Res., Sect. A 250, 125 (1986).

[16] B. A. Peterson, O. D. Oniku, W. C. Patterson, D. Le Roy, A. Garraud, F. Herrault, N. M. Dempsey, D. P. Arnold, and M. G. Allen, Technology development for short-period magnetic undulators, Phys. Procedia 52, 36 (2014).

[17] H. Leupold, Triangular section permanent magnetic structure (1991), https://patents.google.com/patent/ US5014028.

[18] D. C. Quimby, A. L. Pindroh, and K. E. Robinson, The wedged-pole concept for improving the performance of permanent magnet hybrid undulators, Nucl. Instrum. Methods Phys. Res., Sect. A 259, 304 (1987).

[19] A. A. Varfolomeev, A. H. Hairetdinov, S. N. Ivanchenkov, A. S. Khlebnikov, and N.S. Osmanov, Undulator scheme providing wide range magnetic field tuning, Nucl. Instrum. Methods Phys. Res., Sect. A 341, 459 (1994).

[20] O. Chubar, P. Elleaume, and J. Chavanne, RADIA (1997), http://www.esrf.eu/Accelerators/Groups/InsertionDevices/ Software/Radia.

[21] J. A. Clarke, The science and technology of undulators and wigglers (Oxford University Press, New York, 2004), https://doi.org/10.1093/acprof:oso/9780198508557 .001 .0001 .

[22] H. Wang, P. Bencok, P. Steadman, E. Longhi, J. Zhu, and Z. Wang, Complete polarization analysis of an APPLE II undulator using a soft X-ray polarimeter, J. Synchrotron Radiat. 19, 944 (2012).

[23] A. Temnykh, T. Kobela, A. Lyndaker, J. Savino, E. Suttner, and Y. Li, Compact undulator for cornell high energy synchrotron source, IEEE Trans. Appl. Supercond. 22, 4100504 (2012).

[24] C. C. Wright, R. A. Stuart, J. Lucas, and A. Al-Shamma'A, Low cost undulator magnets for industrial free electron masers, Opt. Commun. 185, 387 (2000).

[25] Q. Jia, Effect of undulator harmonics field on free-electron laser harmonic generation, Phys. Rev. Accel. Beams 14, 060702 (2011).

[26] B. M. Kincaid, Analysis of field errors in existing undulators, Nucl. Instrum. Methods Phys. Res., Sect. A 291, 363 (1990).

[27] P. Musumeci, C. Pellegrini, and J. B. Rosenzweig, Higher harmonic inverse free-electron laser interaction, Phys. Rev. E 72, 016501 (2005).

[28] J. B. Rosenzweig et al. (to be published). 